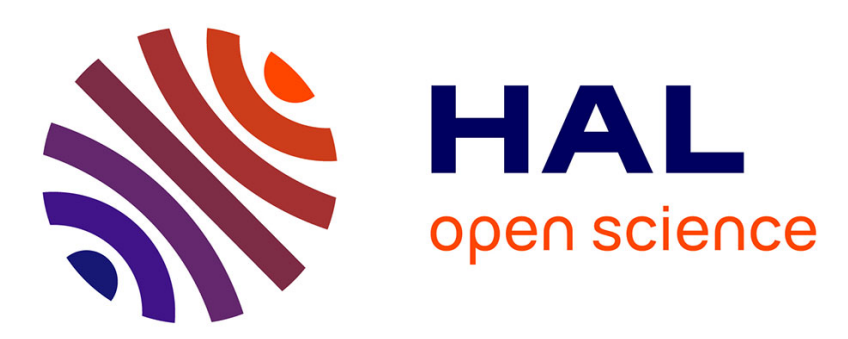

\title{
Imaging multiple local changes in heterogeneous media with diffuse waves
}

Thomas Planes, Éric Larose, Vincent Rossetto, Ludovic Margerin

\section{To cite this version:}

Thomas Planes, Éric Larose, Vincent Rossetto, Ludovic Margerin. Imaging multiple local changes in heterogeneous media with diffuse waves. Journal of the Acoustical Society of America, 2015, 137 (2), pp.660-667. 10.1121/1.4906824 . hal-02376174

\section{HAL Id: hal-02376174 \\ https://hal.science/hal-02376174}

Submitted on 1 Oct 2021

HAL is a multi-disciplinary open access archive for the deposit and dissemination of scientific research documents, whether they are published or not. The documents may come from teaching and research institutions in France or abroad, or from public or private research centers.
L'archive ouverte pluridisciplinaire $\mathbf{H A L}$, est destinée au dépôt et à la diffusion de documents scientifiques de niveau recherche, publiés ou non, émanant des établissements d'enseignement et de recherche français ou étrangers, des laboratoires publics ou privés. 


\title{
Imaging multiple local changes in heterogeneous media with diffuse waves
}

\author{
Thomas Planès ${ }^{\text {a) }}$ and Eric Larose \\ ISTerre Université de Grenoble 1 and CNRS, 38041 Grenoble, France \\ Vincent Rossetto \\ Université de Grenoble 1, LPMMC/CNRS, 25 avenue des Martyrs, 38042 Grenoble, France \\ Ludovic Margerin \\ Université de Toulouse, IRAP/CNRS, 31400 Toulouse, France
}

(Received 26 March 2014; revised 30 October 2014; accepted 8 December 2014)

\begin{abstract}
This study focuses on imaging local changes in heterogeneous media. The method employed is demonstrated and validated using numerical experiments of acoustic wave propagation in a multiple scattering medium. Changes are simulated by adding new scatterers of different sizes at various positions in the medium, and the induced decorrelation of the diffuse (coda) waveforms is measured for different pairs of sensors. The spatial and temporal dependences of the decorrelation are modeled through a diffuse sensitivity kernel, based on the intensity transport in the medium. The inverse problem is then solved with a linear least square algorithm, which leads to a map of scattering cross section density of the changes. (C) 2015 Acoustical Society of America.
\end{abstract}

[http://dx.doi.org/10.1121/1.4906824]

[OU]

Pages: 660-667

\section{INTRODUCTION}

Classical imaging techniques are based on the arrival times of the direct or singly scattered waves. These techniques are extensively used in many different areas (medical imaging, nondestructive testing of materials, seismic imaging) with different types of waves (electromagnetic, acoustic, elastic) at very different scales ( $\mathrm{mm}$ to $\mathrm{km}$ ).

However, in highly heterogeneous media, direct or singly scattered waves are strongly attenuated and these techniques are likely to fail. Strong heterogeneities are common in natural structures (volcanoes, fault zones), as well as in man-made structures (concrete, composite materials), and the difficulty to monitor them is an important concern (Anugonda et al., 2001; Wegler and Lühr, 2001).

The scattering mean free path $\ell$ is the distance that quantifies the degree of heterogeneity of a material and is related to the distance between two successive scattering events. The higher the degree of heterogeneity, the shorter the mean free path. This mean free path is defined as the characteristic length of exponential attenuation of the coherent wave in absence of intrinsic absorption. The coherent wave is the wave that resists ensemble averaging (when such an averaging is practically possible). The energy of the direct wave is not lost, but distributed to multiply scattered waves, also called coda waves, that follow very complex trajectories. Indeed, their propagation paths are similar to random walks and the intensity transport can be described at first order by the solution of the diffusion equation. The growth of the diffusive halo is controlled by the transport mean free path $\ell^{\star}$ that is

\footnotetext{
a) Author to whom correspondence should be addressed. Present address: Colorado School of Mines, Department of Civil and Environmental Engineering, Golden, CO. Electronic mail: tplanes@mines.edu
}

equal to the scattering mean free path when the scattering is isotropic. One usually says that the transport mean free path is the distance after which the wave has lost the memory of its initial direction of propagation.

When the probed medium has a size smaller than or of the order of $\ell$, the single scattering regime applies and most communication, imaging, and monitoring techniques work fine. When the degree of heterogeneity of the medium increases, the scattering mean free path becomes smaller than the size of the probed medium and the waves are scattered multiple times along their trajectories. The so-called "mesoscopic" regime is reached and multiple scattering is observed (Akkermans, 2007). This study focuses on this regime, which concerns strongly heterogeneous media and where standard techniques that work in the single scattering regime are inefficient. The reader can refer to the review of Lagendijk and Van Tiggelen (1996) for a global picture of multiple scattering phenomena.

Imaging beyond a few scattering mean free paths has been demonstrated by techniques aiming at filtering out multiply scattered events to denoise the coherent waves (Borcea et al., 2006; Aubry and Derode, 2009; Rakotonarivo et al., 2011). Our approach is fundamentally different as we try to take advantage of the multiply scattered waves instead of filtering them out. The present work deals with a simulated heterogeneous medium where the distances between sensors range from 5 to $20 \ell^{\star}$. The heterogeneities of the medium are also fixed and averaging over realizations of the disorder is not possible. The coherent waves are not used and even not expected to be retrievable in this case. We note that our method is by essence differential as only changes can be imaged as opposed to defects preexisting in the medium.

Because of their complex trajectories, multiply scattered waves (coda waves) spend much more time in the medium 
than direct or singly scattered waves. Thanks to this property, coda waves show a greater sensitivity to changes occurring in the medium. Using the doublet and stretching technique, very tiny velocity changes have been measured with seismic waves in fault zones (Poupinet et al., 1984; Wegler and Sens-Schönfelder, 2007; Brenguier et al., 2008), volcanoes (Ratdomopurbo and Poupinet, 1995; Grêt et al., 2005; Sens-Schönfelder and Wegler, 2006), and reservoirs (Meunier et al., 2001). These techniques have been formalized and developed under the denomination of coda wave interferometry or CWI (Snieder, 2006). The CWI has been later applied to concrete where minute changes have been monitored using ultrasound (see the review of Planès and Larose, 2013). Likewise, using the sensitivity of coda waves, the diffusing (acoustic) wave spectroscopy technique (Pine et al., 1988; Page et al., 2000; Cowan et al., 2002) allows one to monitor collective movements of scatterers in a fluid medium.

These techniques are designed to monitor global changes taking place in the medium. But, locating/imaging small defects appearing in these media remains a big challenge. During the last 20 years, few techniques have been proposed to solve this problem, most of them using the fluctuations of the intensity speckle (Feng and Sornette, 1991; Nieuwenhuizen and van Rossum, 1993; Vanneste et al., 1993; van Rossum and Nieuwenhuizen, 1999).

Recently, a new method was introduced to locate one small structural change in a multiple scattering medium using the decorrelation of coda waves. This technique was demonstrated with an ultrasound experiment on a concrete block (Larose et al., 2010). It relies on (i) the measurement of the decorrelation of coda waves, (ii) a diffuse sensitivity kernel, and (iii) a $\chi^{2}$ grid-search type inversion. The technique was initially limited to the location of one single, local defect appearing at a time. A detailed study of the forward problem, i.e., how to relate the decorrelation of coda waves to the characteristics of the change (position, cross section) was recently addressed in Planès et al. (2014).

To match real life situations, the method should be able to locate several defects appearing simultaneously and/or spatially extended defects. In a volcano, changes like magma injection may take place simultaneously at several distinct locations in the edifice (Obermann et al., 2013). As for ultrasound, the growth of a crack in concrete under stress may be spatially extended compared to the wavelength.

We dedicate the present study to the following problem: how to locate several local changes appearing at the same time? In the case of velocity changes, a classic linear least square inversion algorithm was used by Froment (2011) to compute a map of velocity changes induced by the 2008 Wenchuan earthquake in China. By weak changes, we imply a relative velocity variation of up to a few percent. Here, we adapt this algorithm (Tarantola and Valette, 1982) to the case of several structural changes, i.e., strong changes corresponding to velocity variations of several tens or hundreds of percents. Contrary to weak velocity changes, structural changes induce a modification of coda waves that cannot be interpreted as a time-shift of the arrivals, but as a waveform distortion or decorrelation. The decorrelation is related to the scattering cross section of the changes through a linear approximation.

We perform controlled numerical experiments to validate the method, as well as the underlying linear approximation. This work also supports the recent applications of the method to real seismic data (Obermann et al., 2013; Obermann et al., 2014).

In Sec. II, we introduce the numerical simulation parameters and configurations. We also introduce the procedure to measure the decorrelation of coda waves, as well as the expression of the theoretical sensitivity kernel. In Sec. III, we reformulate the forward problem to describe several local changes before detailing and validating linear least-square inversions.

\section{DECORRELATION OF CODA WAVES}

This section follows closely the first section of Planès et al. (2014), as we use similar numerical simulations and measurements. We refer the reader to the original publication for additional details on the numerical aspects.

\section{A. Simulation parameters}

To simulate the multiple scattering of waves in heterogeneous media, we perform numerical experiments of acoustic wave propagation in a medium with constant background velocity, filled with thousands of impenetrable point scatterers. To solve the wave equation of the pressure field, we use a classic second order two-dimensional (2D) finite difference scheme (Taflove and Hagness, 2005). In the following, temporal (respectively, spatial) quantities are expressed in units of $\tau_{0}$ (respectively, $\lambda_{0}$ ), the central period (respectively, wavelength) of the source wavelet. We consider a square shaped lossless medium with reflective boundaries (Dirichlet boundary condition) of length $L=200 \lambda_{0}$, constant velocity $c_{0}=\lambda_{0} / \tau_{0}$, in which a point source sends a Gaussian impulse of central frequency $f_{0}=1 / \tau_{0}$ and relative bandwidth $\Delta f / f_{0}=30 \%$ (at $\left.-3 \mathrm{~dB}\right)$. The spatial gridstep is $a=\lambda_{0} / 15$ and the time step of the scheme is set to $\eta=a /\left(c_{0} \sqrt{2}\right)$ $\simeq 0.0471 \tau_{0}$, based on the Courant-Friedrichs-Lewy stability condition (Courant et al., 1967).

We choose to work within the independent scattering approximation that concerns dilute systems, i.e., where the scattering mean free path is much larger than the central wavelength, $\ell \gg \lambda_{0}$. This means that the correlations between scatterers are negligible and that the different scattering paths do not interfere with one another. The intensity transport can then be described as the sum of the intensities along each scattering path. This allows us to use analytic intensity propagators to build the later introduced sensitivity kernels. We determine the number of scatterers in the medium so that $\ell=10 \lambda_{0}$. Each soft scatterer is defined by a null-pressure condition at its grid point. As only one grid point is modified for each scatterer, they do not have a defined geometry, but are much smaller than the wavelength $(\sim \lambda / 15)$. We evaluated the scattering cross section of these point scatterers, $\sigma=0.174 \lambda_{0}$, through additional simulations. To do so, only one of these scatterers was placed in a 
homogeneous medium and the scattered energy from an incident plane wave was evaluated.

The number $N$ of scatterers to place in the medium is then deduced from the usual relation $\ell=1 / n \sigma$, where $n=N / S$ is the density of scatterers and $S=L^{2}$ is the surface of the $2 \mathrm{D}$ medium. The scatterers are randomly positioned in space and uncorrelated. Given their small size compared to the wavelength, the scattering and transport cross sections of the scatterers are similar $\left(\sigma \simeq \sigma^{\star}\right)$. This means that the scattering is isotropic, and it also implies the equality of the scattering and transport mean free paths $\left(\ell \simeq \ell^{\star}\right)$. All the relevant simulation parameters are summarized in Table I.

A typical realization of the random medium is shown in Fig. 1(a) where the gray dots represent the point scatterers. We arbitrarily place ten sensors in the medium that can act either as a source or a receiver. In addition, three distinct locations have been arbitrarily selected in the medium to host the apparition of additional soft scatterers that we wish to image. These defects appearing at locations $A, B$, and $C$ are disks of respective radius $0.2,0.33$, and $0.5 \lambda_{0}$. We insist on the fact that the background scattering medium remains unchanged throughout the study, as it would be the case for real solid heterogeneous structures.

As an example, the impulse response $\phi^{2,7}$ between sensors 2 and 7 is displayed in Fig. 1(b). The impulse response shows long-lasting wave trains constituted of multiply scattered waves. Since the medium is lossless and bounded with perfectly reflective boundaries, the amplitude of the coda stabilizes after sufficient time without any decay.

\section{B. Physical model of the decorrelation}

To study the variations of the waveforms induced by local changes in the medium, we compare impulse responses acquired before and after the change(s). To simulate local structural changes, we introduce a new scatterer in the medium at one (or several) of the three candidate locations selected [Fig. 1(a)]. At short lapse-times, the waveforms acquired before and after the change are nearly identical to each other. However, at long times, we observe slight differences in phase and amplitude of the coda waveforms. The addition of a defect results in a distortion or decorrelation of the waveforms.

TABLE I. Dimensionless value of the simulation parameters.

\begin{tabular}{lcc}
\hline \hline Notation & $\begin{array}{c}\text { Dimensionless } \\
\text { value }\end{array}$ & Description \\
\hline$f_{0}$ & $1 / \tau_{0}$ & Central frequency \\
$c_{0}$ & $\lambda_{0} / \tau_{0}$ & Wave speed \\
$\lambda_{0}$ & $\lambda_{0}$ & Central wavelength \\
$\Delta f / f_{0}$ & $30 \%$ & Relative bandwidth \\
$a$ & $\lambda_{0} / 15$ & Spatial grid step \\
$\eta$ & $0.0471 \tau_{0}$ & Temporal step \\
$L$ & $200 \lambda_{0}$ & Medium length \\
$\sigma_{0} \simeq \sigma_{0}^{\star}$ & $0.174 \lambda_{0}$ & Scattering and transport cross section \\
& & of a point scatterer \\
$\ell \simeq \ell^{\star}$ & $10 \lambda_{0}$ & Scattering and transport mean free path \\
$N$ & 23000 & Number of point scatterers in the medium \\
\hline \hline
\end{tabular}
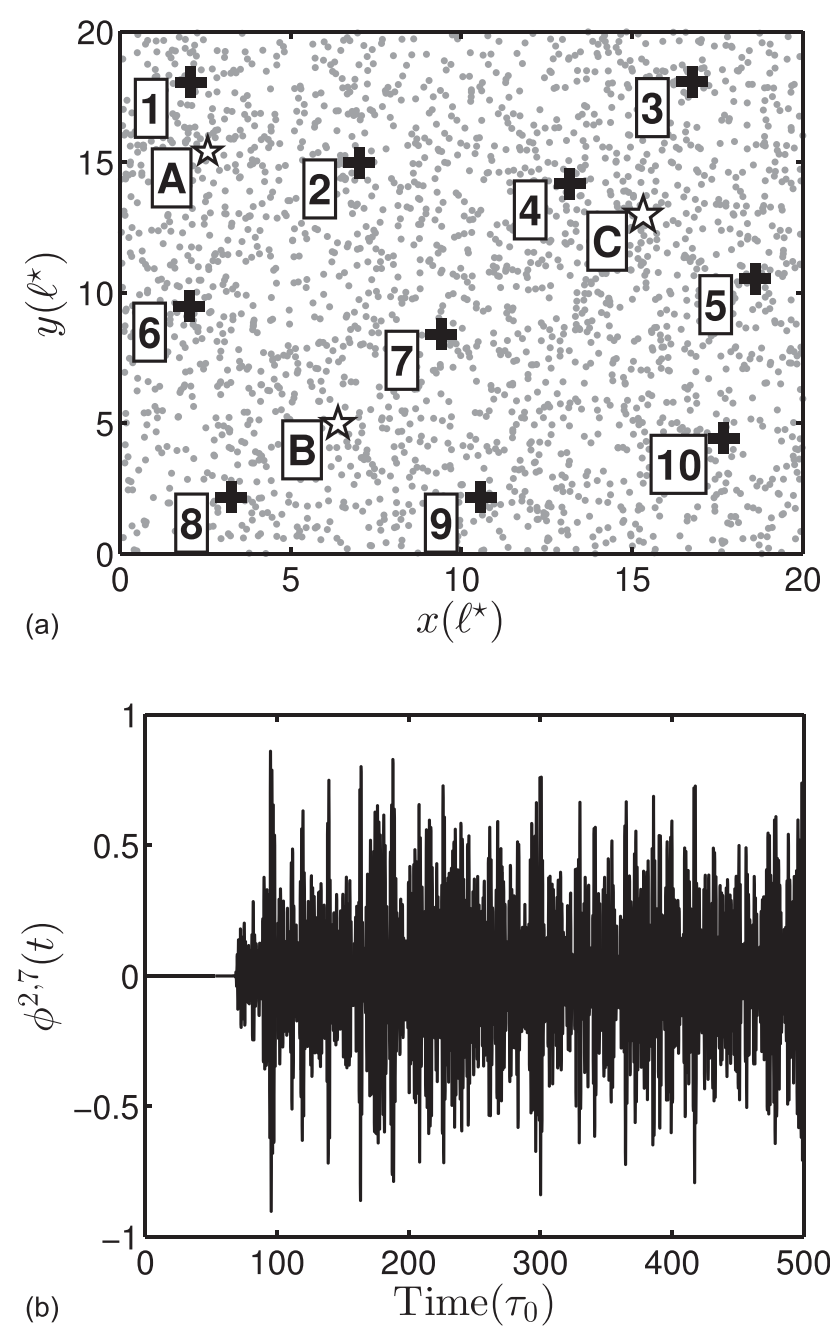

FIG. 1. (a) Example of a heterogeneous, disordered medium. The gray dots represent the point scatterers, the crosses show the sensors, and the stars are the three locations of the appearing defects. (b) The impulse response between sensors 2 and 7 shows a long tail (coda) constituted by multiply scattered waves.

To quantify the change between an initial state of impulse response $\phi_{0}(t)$ and a final state of impulse response $\phi_{1}(t)$ the experimental decorrelation coefficient is defined as

$$
D C^{\operatorname{Exp}}(t)=1-\frac{\int_{t-T / 2}^{t+T / 2} \phi_{0}\left(t^{\prime}\right) \phi_{1}\left(t^{\prime}\right) d t^{\prime}}{\sqrt{\int_{t-T / 2}^{t+T / 2} \phi_{0}\left(t^{\prime}\right)^{2} d t^{\prime} \int_{t-T / 2}^{t+T / 2} \phi_{1}\left(t^{\prime}\right)^{2} d t^{\prime}}} .
$$

The decorrelation corresponds to one minus the normalized correlation coefficient. It is calculated in a time window of length $T$ and can be evaluated at different times $t$ in the coda. The size of the time window is typically a few tens of central periods $\tau_{0}$.

The decorrelation induced by the defect $C$ for different pairs of sensors is shown in Fig. 2. We observe that the decorrelation always increases with the time in the coda. Indeed, the longer the wave paths, the higher the chances to interact with the change, which results in more pronounced waveform distortions. Another key observation is that the 


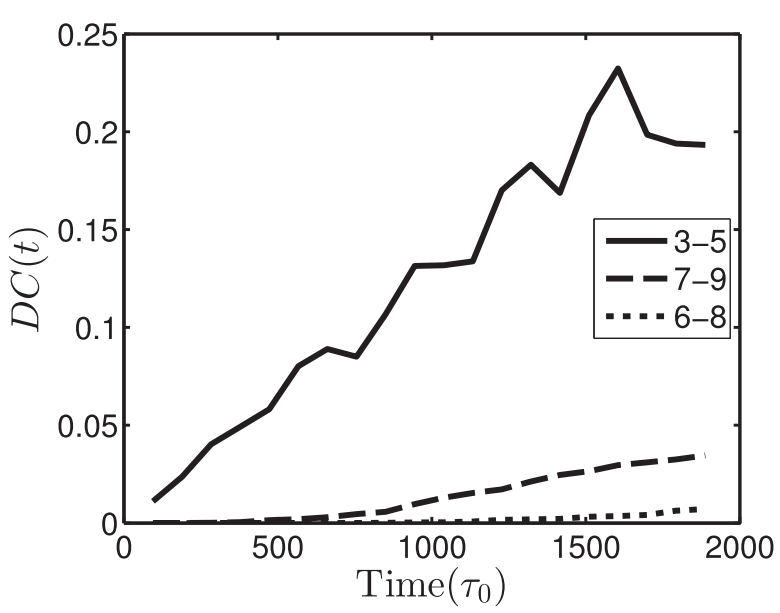

FIG. 2. Decorrelation of coda waves induced by the addition of defect $C$ for different pairs of sensors. For each pair of sensor, the decorrelation $D C$ increases with coda time $t$. The closer the defect to the sensors line, the higher the decorrelation: $D C_{3,5}>D C_{7,9}>D C_{6,8}$.

decorrelation strongly depends on the position of the change relative to the position of the source and receiver: the closer the defect to the source-receiver line, the stronger the variation of the waveforms.

When dealing with noisy data, the decorrelation is likely to be overestimated using the expression above. A baseline decorrelation level only due to noise can be estimated from repetitive acquisitions of impulse responses during a quiet period, i.e., when the medium does not change. This baseline level is then deduced from the actual decorrelation measurements to minimize the impact of noise on the method (Obermann et al., 2013).

In previous studies (Rossetto et al., 2011; Planès et al., 2014), we showed using diagrammatic techniques that the decorrelation can be theoretically estimated as

$$
\begin{aligned}
& D C^{\mathrm{Th}}(\boldsymbol{r}, t)=\frac{c \sigma}{2} K(\boldsymbol{S}, \boldsymbol{R}, \boldsymbol{r}, t), \\
& K(\boldsymbol{S}, \boldsymbol{R}, \boldsymbol{r}, t)=\frac{\int_{0}^{t} p(\boldsymbol{S}, \boldsymbol{r}, u) p(\boldsymbol{r}, \boldsymbol{R}, t-u) d u}{p(\boldsymbol{S}, \boldsymbol{R}, t)},
\end{aligned}
$$

where $\sigma$ is the scattering cross section of the new defect, $c$ is the wave velocity, and $K$ is a sensitivity kernel based on the intensity propagator $p(\boldsymbol{a}, \boldsymbol{b}, t)$ between $\boldsymbol{a}$ and $\boldsymbol{b}$. The symbols $\boldsymbol{S}, \boldsymbol{R}$, and $\boldsymbol{r}$ denote the position of the source, receiver, and defect, respectively, and $t$ is the time in the coda.

In strongly heterogeneous media, the intensity transport can be described by different approximations as the diffusion equation or the more accurate radiative transfer equation (Ryzhik et al., 1996). For the case of isotropic scattering in 2D infinite media, an exact solution of the radiative transfer equation has been derived independently by Shang and Gao (1988), Sato (1993), and Paasschens (1997). The expression for the intensity propagator reads

$$
\begin{aligned}
p(r, t)= & \frac{e^{-r / \ell}}{2 \pi r c} \delta\left(t-\frac{r}{c}\right)+\frac{1}{4 \pi D t}\left(1-\frac{r^{2}}{c^{2} t^{2}}\right)^{-1 / 2} \\
& \times e^{\left(\sqrt{c^{2} t^{2}-r^{2}}-c t\right) / \ell} \Theta(c t-r)
\end{aligned}
$$

where the isotropic scattering implies the equality of the scattering and transport mean free paths, $\ell=\ell^{\star}$. The diffusion constant is $D=c \ell^{\star} / 2$ and the Heaviside function, $\Theta$, ensures the causality of the solution (the energy cannot travel faster than the wave velocity). This solution is composed of the coherent intensity term, decaying exponentially with the mean free path, and a diffuse term that includes all the scattering orders. It is, thus, also valid at short times and distances and one can verify that this solution converges toward the diffusion solution for times $t \gg \ell / c$.

We use this solution along with the method of images to take into account the reflections of the wave energy on the boundaries. The method of images describes the total intensity as the sum of the infinite medium intensity of the real source plus the infinite medium intensities of virtual sources. The positions of the virtual sources are obtained as mirror images of the real source from the boundaries of the medium (Crank, 1979).

The decorrelation is proportional to the ratio of the intensity of the waves that interacted with the defect in $r$ over the total intensity propagated from $\boldsymbol{S}$ to $\boldsymbol{R}$. The decorrelation is shown to depend on the spatial location and scattering cross section of the change, as well as on the coda time where the measurements are performed. Our imaging technique relies on these key properties.

The expression (2) holds when a single defect is appearing in the medium. The extension of this relation to several defects appearing at the same time is discussed in Sec. III A.

\section{WAVEFORM DECORRELATION: THE INVERSE PROBLEM}

The problem of locating one local structural change has been addressed by Larose et al. (2010) and Rossetto et al. (2011) using ultrasound experiments in a concrete block, as well as numerical simulations. The inverse method employed in these studies consisted of a grid search of the location of the change using a $\chi^{2}$ cost function. However, this method is unable to locate several simultaneous changes (Planès, 2013). In Sec. III A, we reformulate the forward problem and in Sec. III B, we adapt a linear least square inversion method to locate simultaneous structural changes (Tarantola and Valette, 1982; Tarantola, 2005).

\section{A. Reformulation of the forward problem}

On a theoretical level, the decorrelation has only been studied in the case of a single and local change appearing in the medium (Rossetto et al., 2011; Planès et al., 2014). In practice, several or extended changes can occur simultaneously in structures that one wishes to monitor: cracks in concrete, magma intrusion in a volcano, active fault slip, etc.

When the change is a weak velocity variation, the main effect on coda waves is not a decorrelation, but a phase shift. In this case, the forward problem can be linearly adapted to multiple and extended defects (Pacheco and Snieder, 2005; Planès et al., 2014). In general, this is not the case for the decorrelation induced by structural changes. 
Here, we do not tackle the problem of extended defects, but we focus on the simultaneous apparition of distinct local defects. If these defects are weak and distant enough, we assume that we may neglect their interactions and formulate the effect of their apparition as a linear superposition of their individual effect. This assumption of linearity is a significant approximation that will be validated a posteriori.

Let us discretize the medium into $P$ voxels of volume, $\delta V$. To each voxel $i$ centered on the position $\boldsymbol{r}_{i}$ is associated a scattering cross section density $\Sigma_{i}$. $\Sigma_{i}$ corresponds to the scattering cross section of the change taking place in the voxel $i$ divided by the voxel volume $\delta V$. The change at each point of the medium is thus quantified by a column vector $\Sigma$ of length $P$, where the density of scattering cross section is stored. Similarly, the $N$ measured decorrelations (different pairs of sensors and different coda times) are gathered in a column vector $\boldsymbol{D C}$ of length $N$. These two vectors can then be related through a discretized version of the kernel $K$ [Eq. (2)], as a $N \times P$ matrix $K_{i j}=K\left(\boldsymbol{S}_{\boldsymbol{i}}, \boldsymbol{R}_{\boldsymbol{i}}, \boldsymbol{r}_{\boldsymbol{j}}, t_{i}\right) . \boldsymbol{S}_{\boldsymbol{i}}, \boldsymbol{R}_{\boldsymbol{i}}$, and $t_{i}$ are the source position, receiver position, and center coda time of the measurement $i$, respectively, and $\boldsymbol{r}_{j}$ is the central position of voxel $j$. The linear relation between $\boldsymbol{D C}$ and $\boldsymbol{\Sigma}$ is written as a matrix product, which is an extension of the relation (2) to multiple changes

$$
D C=\frac{c \delta V}{2} \boldsymbol{K} \Sigma
$$

where $c$ is the wave velocity. There exists a large variety of methods to solve linear inverse problems (Nocedal and Wright, 1999) of the form

$$
\boldsymbol{d}=\boldsymbol{G m},
$$

where $\boldsymbol{d}$ are the measured data (here $\boldsymbol{D} \boldsymbol{C}^{\mathbf{E x p}}$ ), $\boldsymbol{m}$ is model that we wish to estimate (here $\boldsymbol{\Sigma}$ ), and $\boldsymbol{G}$ is the physics that relates them [here $(c \delta V / 2) \boldsymbol{K}]$. We detail the application of a least square algorithm in Sec. III B.

\section{B. Inversion algorithm}

In this work, we adopt a least square method (Tarantola and Valette, 1982; Tarantola, 2005) that leads to an explicit solution through the inversion of matrices. Let us suppose that we have a priori knowledge of the model $\boldsymbol{m}_{\text {prior }}$. We also suppose that we know the covariance matrix of the model $\boldsymbol{C}_{\boldsymbol{M}}$ and the covariance matrix of the data $\boldsymbol{C}_{\boldsymbol{D}}$. A least square misfit function $S(\boldsymbol{m})$ is constructed as

$$
\begin{aligned}
\mathrm{S}(\boldsymbol{m})= & \left(\boldsymbol{G} \boldsymbol{m}-\boldsymbol{d}_{\mathrm{obs}}\right)^{T} \boldsymbol{C}_{\boldsymbol{D}}^{-1}\left(\boldsymbol{G} \boldsymbol{m}-\boldsymbol{d}_{\mathrm{obs}}\right) \\
& +\left(\boldsymbol{m}-\boldsymbol{m}_{\text {prior }}\right)^{T} C_{\boldsymbol{M}}^{-1}\left(\boldsymbol{m}-\boldsymbol{m}_{\text {prior }}\right) \\
= & \left\|\boldsymbol{G} \boldsymbol{m}-\boldsymbol{d}_{\mathrm{obs}}\right\|_{D}^{2}+\left\|\boldsymbol{m}-\boldsymbol{m}_{\text {prior }}\right\|_{M}^{2},
\end{aligned}
$$

where $\|\cdot\|_{D}^{2}$ and $\|\cdot\|_{M}^{2}$ are $\ell_{2}$ norms weighted by $\boldsymbol{C}_{\boldsymbol{D}}^{-1}$ and $\boldsymbol{C}_{\boldsymbol{M}}^{-1}$, respectively. The cost function is composed of two terms: the distance between the estimated model and the one known a priori, and the distance between the measured data and the response of the estimated model. The covariance matrices $\boldsymbol{C}_{\boldsymbol{M}}$ and $\boldsymbol{C}_{\boldsymbol{D}}$ allow us to give weight to each term according to the confidence in the a priori model and in the measured data. In addition, the nondiagonal elements of these matrices allow us to introduce correlations between data and/or cells of the model.

According to Tarantola (2005), the estimated model $\tilde{\boldsymbol{m}}$ that minimizes the cost function writes

$$
\tilde{\boldsymbol{m}}=\boldsymbol{m}_{\text {prior }}+\left(\boldsymbol{G}^{T} \boldsymbol{C}_{\boldsymbol{D}}^{-1} \boldsymbol{G}+\boldsymbol{C}_{\boldsymbol{M}}^{-1}\right)^{-1} \boldsymbol{G}^{T} \boldsymbol{C}_{\boldsymbol{D}}^{-1}\left(\boldsymbol{d}_{\mathrm{obs}}-\boldsymbol{G} \boldsymbol{m}_{\text {prior }}\right)
$$

with the a posteriori covariance given by

$$
\tilde{\boldsymbol{C}}_{M}=\left(\boldsymbol{G}^{T} \boldsymbol{C}_{\boldsymbol{D}}^{-1} \boldsymbol{G}+\boldsymbol{C}_{M}^{-1}\right)^{-1} .
$$

We now detail the construction of the covariance matrices and the application of the inversion algorithm to our numerical experiment where three defects $A, B$, and $C$, appear simultaneously in the medium. We used $N=566$ measurements corresponding to the 45 possible distinct pairs of sensors and an average of $\sim 12$ non-overlapping measurement time windows per sensor pair.

The covariance matrix $\boldsymbol{C}_{\boldsymbol{D}}$ describes the correlations and errors on the measured data while the covariance matrix $\boldsymbol{C}_{\boldsymbol{M}}$ corresponds to the expected deviations of the real model from the one known a priori, as well as its correlations. The construction and tuning of these matrices is the key point for the success of the inversion.

For the sake of simplicity, we neglect the correlations between the different measurements, making $\boldsymbol{C}_{\boldsymbol{D}}$ a diagonal matrix. This assumption is reasonable for distant sensors and non-overlapping measurement time windows. We also assume that the relative error is the same for all measurements. We note that a theoretical estimator of the measurement error still needs to be developed. Estimating this error from the actual measurements is challenging because it would require having access to different realizations of the disorder, which is not the case in practice.

As a workaround, we compare decorrelation measurements performed in neighboring time windows, and roughly evaluate the relative fluctuations of the measurements to be $\sim 30 \%$. Under the assumptions made, we actually notice that this value can be arbitrary, as only the relative weight of the matrices $\boldsymbol{C}_{\boldsymbol{M}}$ and $\boldsymbol{C}_{\boldsymbol{D}}$ influences the minimization of the misfit function $S(\boldsymbol{m})$. The matrix $\boldsymbol{C}_{\boldsymbol{D}}$ is defined as

$$
C_{D i j}=\left(0.3 d_{i}\right)^{2} \delta_{i j}
$$

where $\delta_{i j}$ is the Kronecker symbol and $d_{i}$ is the measurement $i$. To reduce the under-determination of the problem, we define an exponential correlation between the model cells, following Froment (2011),

$$
C_{M i j}=\left(\sigma_{m} \frac{L_{0}}{L_{c}}\right)^{2} e^{-\left\|r_{i}-r_{j}\right\| / L_{c}},
$$

where $\sigma_{m}$ is a standard deviation from the a priori model $\boldsymbol{m}_{\text {prior }}, L_{0}=8 \lambda_{0}$ corresponds to the arbitrary linear size of a model cell, chosen as a trade-off between resolution and computation time, and $L_{c}$ is a typical correlation distance between cells. 
The inversion algorithm thus involves two free parameters $L_{c}$ and $\sigma_{m}$ to be tuned. We follow the $L$-curve method described by Hansen (1992), which allows one to tune the free parameters as a trade-off between the quality of the fit $\left\|\boldsymbol{d}_{\text {obs }}-\boldsymbol{G} \tilde{\boldsymbol{m}}\right\|$ and a norm of the estimated model $\tilde{\boldsymbol{m}}$.

Having no a priori information on the positions or strength of the expected changes, we start with a null $a$ priori model $\boldsymbol{m}_{\text {prior }}=\mathbf{0}$. In regard to the range of validity of the method, we suppose that the medium hardly changes between the two series of acquisitions. To constrain the inversion result to be simple, we choose to consider the $\ell_{1}$ norm of the distance between the estimated model and the $a$ priori model

$$
\ell_{1}(\tilde{\boldsymbol{m}})=\sum_{i}\left|\tilde{m}_{i}-m_{\text {prior }_{i}}\right| .
$$

The a priori model being null, the norm is the $\ell_{1}$ norm of the estimated model.

To plot the $L$-curve, we define a range of potential values for the two free parameters, $L_{c}=2-25 \lambda_{0}$ and $\sigma_{m}=1 \times 10^{-4}-1 \lambda_{0} / \ell^{* 2}$, and perform as many inversions as possible combinations of these values. For each inversion, we calculate the corresponding residual $\left\|\boldsymbol{d}_{\text {obs }}-\boldsymbol{G} \tilde{\boldsymbol{m}}\right\|$ and the $\ell_{1}$ norm of the model. The $L$-curve is a parametric representation of the residual versus the $\ell_{1}$ norm. An $L$-curve example is represented on Fig. 3 for $L_{c}=16 \lambda_{0} \approx 1.5 \ell^{\star}$ ( $\sigma_{m}$ free). Only one free parameter is shown for better visualization. $L$-curves obtained for different $L_{c}$ values actually show very similar behaviors and the choice of a particular $L_{c}$ is somewhat arbitrary and allows one to tune the smoothness of the inverted map, i.e., to control the intensity of its spatial fluctuations.

Looking at the cost function composition (6), we note that the tuning of $\sigma_{m}$ allows giving more or less weight to each of the two terms. A high $\sigma_{m}$ gives more importance to the term $\left\|\boldsymbol{G} \boldsymbol{m}-\boldsymbol{d}_{\mathbf{o b s}}\right\|_{D}^{2}$ and privileges the fit of the measured

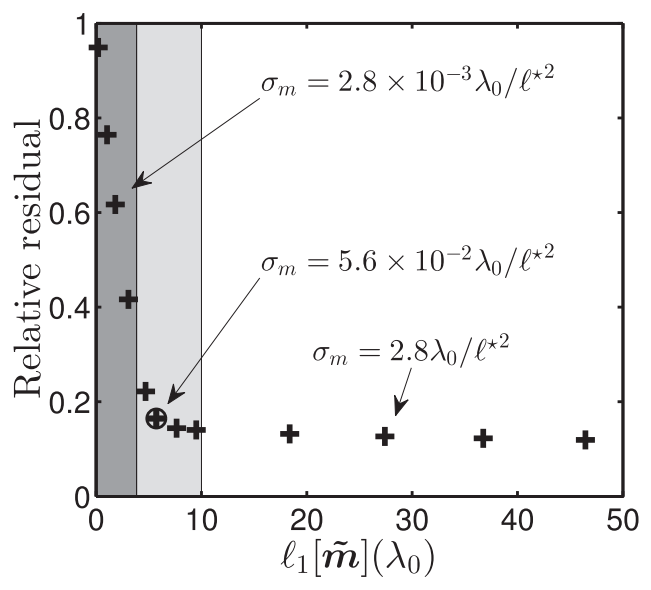

FIG. 3. $L$-curve obtained for a correlation distance $L_{c}=16 \lambda_{0}$. For each tested value of standard deviation from the a priori model $\sigma_{m}$, the relative residual of the inversion is plotted versus the $\ell_{1}$ norm of the estimated model. The dark area shows a high error (high residue), but a simple model (weak $\ell_{1}$ norm). The light area shows a weak error (small residue), but a complex model (high $\ell_{1}$ norm). The gray area in between shows the best trade-off between simplicity of the estimated model and quality of the fit of the measured data. The standard deviation is thus chosen around $\sigma_{m}=5.6$ $\times 10^{-2} \lambda_{0} / \ell^{* 2}$. data (small residue). To the contrary, a weak $\sigma_{m}$ gives more importance to the term $\left\|\boldsymbol{m}-\boldsymbol{m}_{\text {prior }_{i}}\right\|_{M}^{2}$, and privileges the fidelity to the a priori model (weak $\ell_{1}$ norm).

The point of maximum curvature of the $L$-curve allows us to select the free parameter(s) as a trade-off between the simplicity of the estimated model and the quality of the fit (Fig. 3). The simplicity (or complexity) of the estimated model is understood as the intensity of its deviations from the a priori model, as measured by the $\ell_{1}$ norm. The covariance matrix $\boldsymbol{C}_{\boldsymbol{M}}$ is here constructed from the parameters $\sigma_{m}=5.6 \times 10^{-2} \lambda_{0} / \ell^{\star 2}$ and $L_{c}=16 \lambda_{0}$. The inversion algorithm (7) is then applied to obtain the estimated model $\tilde{\boldsymbol{m}}$.

The density of scattering cross section map $\tilde{\boldsymbol{\Sigma}}=\tilde{\boldsymbol{m}}$ obtained is plotted in Fig. 4(a). This map seems to roughly indicate the areas of change, but with a rather weak contrast. In addition, negative cross section areas are present, despite their lack of physical meaning.

The algorithm used here does not include a positivity constraint on the model. To tackle this issue, we propose to iterate the procedure, truncating the negative values of
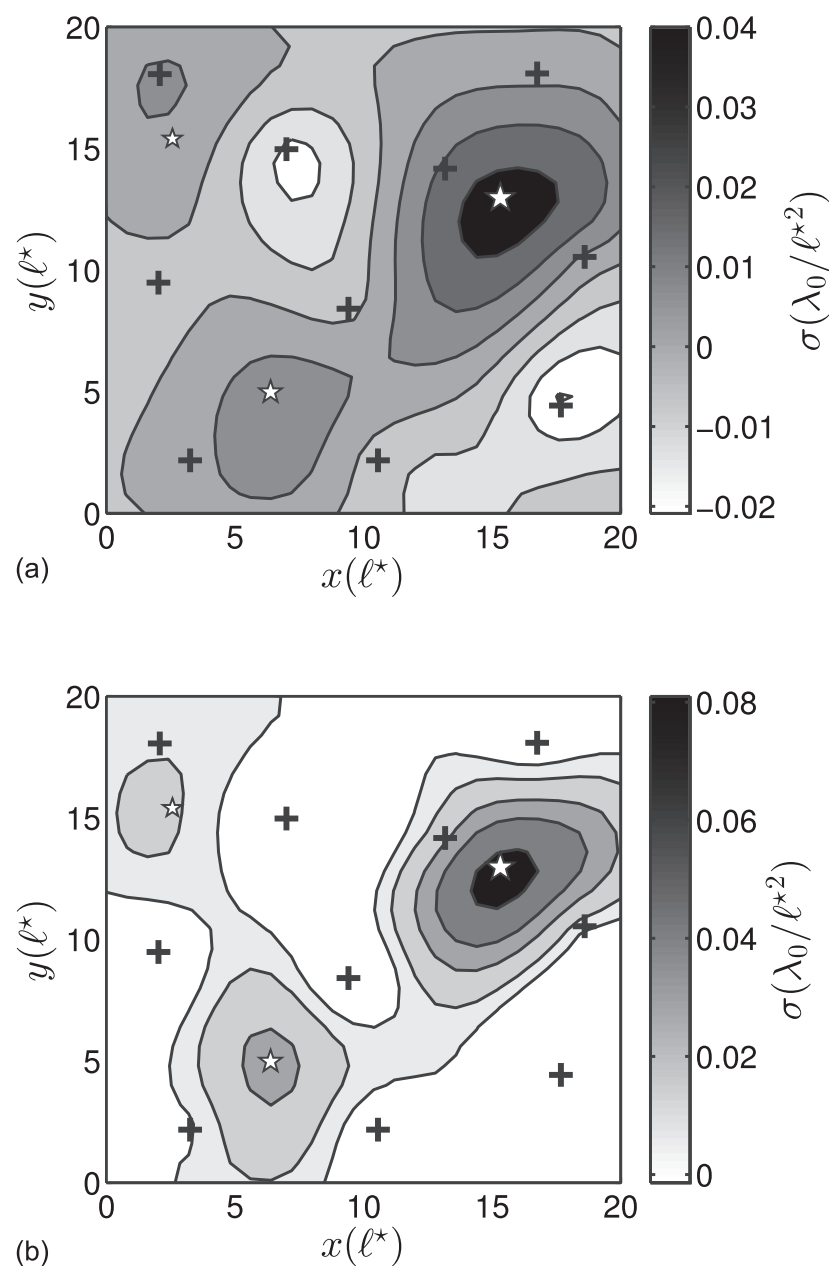

FIG. 4. Scattering cross section density map obtained with the linear least square inversion. The positions of the three defects of radius $r_{s}=\lambda / 5, \lambda / 3, \lambda /$ 2 , are indicated by the stars. (a) Map obtained after a single iteration, the areas encompassing the defects are visible, but the contrast is weak. In addition, areas of negative cross sections (not physical) are found. (b) Map obtained after about ten iterations. The defects are well localized, the positivity constraint is respected, and the scattering cross section density is in qualitative agreement with the size of the defects. 
TABLE II. Real and estimated cross sections of the defects in wavelength unit. The linear least square method uses a single numerical experiment where the three defects appear simultaneously. The cross sections are then estimated by integrating the cross section density in distinct spatial areas.

\begin{tabular}{lccc}
\hline \hline Defect & $\mathrm{A}$ & $\mathrm{B}$ & $\mathrm{C}$ \\
\hline Radius $\left(\lambda_{0}\right)$ & 0.2 & 0.33 & 0.5 \\
$\sigma_{\text {real }}\left(\lambda_{0}\right)$ & 1.05 & 2.49 & 4.49 \\
$\sigma_{\text {inverted }}\left(\lambda_{0}\right)$ & 0.93 & 1.68 & 3.35 \\
Relative error $(\%)$ & 11 & 33 & 25 \\
\hline \hline
\end{tabular}

the model at each step. The model estimated at iteration $n$ is used as the a priori model for the iteration $n+1$. The a posteriori covariance $\tilde{\boldsymbol{C}}_{M}$ [Eq. (8)] at iteration $n$ is also used as the covariance of iteration $n+1$. The applied algorithm has the form of a recurrence relation

$$
\tilde{\boldsymbol{m}}^{n+1}=\tilde{\boldsymbol{m}}_{0}^{n}+\left(\boldsymbol{G}^{T} \boldsymbol{C}_{\boldsymbol{D}}^{-1} \boldsymbol{G}+\tilde{\boldsymbol{C}}_{M}^{n}{ }^{-1}\right)^{-1} \boldsymbol{G}^{T} \boldsymbol{C}_{\boldsymbol{D}}^{-1}\left(\boldsymbol{d}_{\mathbf{o b s}}-\boldsymbol{G} \tilde{\boldsymbol{m}}_{0}^{n}\right),
$$

where $\tilde{\boldsymbol{m}}_{0}^{n}$ is the estimated model at iteration $n$, from which the negative elements have been set to zero. After about ten iterations, the obtained map converges toward the result shown in Fig. 4(b), where all three defects are well located and for which the positivity constraint is respected. We note that a positivity constraint could have been imposed by inverting the logarithm or the square of the model. However, this would have generated a non-linear forward problem and required more advanced inversion techniques.

Because of the spatial smoothing, the cross sections of the defects are spread in large areas around their real positions. To estimate their scattering cross sections, we integrate the scattering cross section densities in disks of radius $5 \ell^{\star}$ around each of the three local maxima. The estimated cross sections are given in Table II. The relative error in the cross section estimation is larger for the bigger defects. This might indicate that the model of the decorrelation that we used starts to be inaccurate for these sizes of defects.

The quality of the inversion can be verified with the resolution operator $\boldsymbol{R}$ defined as (Tarantola and Valette, 1982)

$$
\boldsymbol{R}=\boldsymbol{I}-\tilde{\boldsymbol{C}}_{M} \boldsymbol{C}_{M}^{-1}
$$

For each cell $i$ of the model, the averaging index (Vergely et $a l ., 2010$ ) is given by $\Sigma_{j} R_{i j}$. In the areas where the averaging index is low, the estimated model remains similar to the a priori model, and this independently of the values of the true model in the area. This means that the observed data provides poor information on these areas. To the contrary, when the averaging index is close to 1 , the estimated model corresponds to a spatial average of the true model. The averaging index of each cell of the model is shown in Fig. 5. The averaging index is close to 1 for the whole region covered by the sensors, indicating a good resolution in this area. The quality of the inversion is expected to be low only near the boundaries, outside the instrumented area.

Using this inversion scheme, we located three defects of different sizes that appeared simultaneously at distinct locations. This supports the validity of the underlying linear

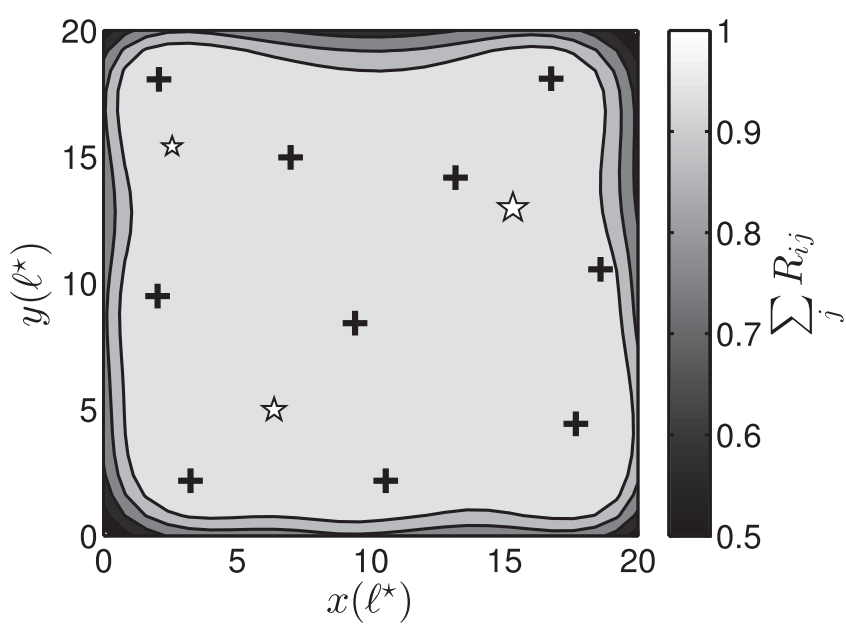

FIG. 5. Map of the averaging index of the resolution operator $\boldsymbol{R}$. The averaging index is very high $(>0.9)$ for the whole region covered by the sensors, indicating a good resolution. The quality of the inversion is expected too be low only near the boundaries where the averaging index drops down to 0.5.

approximation, at least regarding several local and distinct defects. Trying to image extended and/or nearby appearing defects would provide more insights into the regime of validity of this linear approximation.

\section{CONCLUSION}

In this study, we addressed the problem of imaging several defects appearing simultaneously in a multiple scattering medium. Using different pairs of sources and receivers, we measured the decorrelation of the coda waves induced by the new defects. Using a grid-search $\chi^{2}$ technique, previous studies were able to locate one defect appearing at a time, but failed to locate several defects appearing simultaneously.

We reformulated the direct problem, assuming that the effect of several small and distant defects appearing simultaneously may be modeled as a linear combination of their individual effect. To solve this linear problem, we used a linear least square inversion algorithm with regularization parameters. We tuned these free parameters using the $L$-curve method, which expresses the trade-off between the quality of the fit and the simplicity of the estimated model. In addition, we built an iterative procedure to add a positivity constraint on the estimated model.

The estimated model is a map of scattering cross section density that indicates the positions of the defects. The scattering cross sections are also in qualitative agreement with the size of the defects. These results validate the method and the underlying linear approximation.

This work supports the recent applications of the method to monitoring temporal changes on active volcanoes (Obermann et al., 2013) and fault zones (Obermann et al., 2014).

Several questions still need to be addressed. How to model the decorrelation induced by a large defect? Is it possible to image the geometry of such changes? What is the resolving power of the method? Studying more general changes will likely require a reformulation of the direct problem that might no longer be linearly dependent on the 
scattering cross sections of the defects. The problem might possibly be solved using non-linear inversion algorithms. The development of sensitivity kernels for the elastic case is also of great importance for the applications. These kernels should include the transport and conversion of the different propagation modes ( $\mathrm{P}, \mathrm{S}$, and surface waves).

\section{ACKNOWLEDGMENTS}

This work was partially funded by the Agence Nationale de la Recherche JC08-313906 SISDIF grant, and by the Vulnérabilité des Ouvrages aux Risques program. ISTerre is part of the Labex Observatoire des Sciences de l'Univers de Grenoble@2020 (ANR10LABX56).

Akkermans, E. (2007). Mesoscopic Physics of Electrons and Photons (Cambridge University Press, Cambridge, UK), pp. 1-568.

Anugonda, P., Wiehn, J., and Turner, J. (2001). "Diffusion of ultrasound in concrete," Ultrasonics 39, 429-435.

Aubry, A., and Derode, A. (2009). "Detection and imaging in a random medium: A matrix method to overcome multiple scattering and aberration," J. Appl. Phys. 106, 044903.

Borcea, L., Papanicolaou, G., and Tsogka, C. (2006). "Coherent interferometric imaging in clutter," Geophysics 71, SI165-SI175.

Brenguier, F., Campillo, M., Hadziioannou, C., Shapiro, N., Nadeau, R., and Larose, E. (2008). "Postseismic relaxation along the San Andreas fault at Parkfield from continuous seismological observations," Science 321, 1478-1481.

Courant, R., Friedrichs, K., and Lewy, H. (1967). "On the partial difference equations of mathematical physics," IBM J. Res. Dev. 11, 215-234.

Cowan, M., Jones, I., Page, J., and Weitz, D. (2002). "Diffusing acoustic wave spectroscopy," Phys. Rev. E 65, 066605.

Crank, J. (1979). The Mathematics of Diffusion (Oxford Science Publications, New York), pp. 1-414.

Feng, S., and Sornette, D. (1991). "Acoustical nondestructive evaluation of heterogeneous materials in the multiple scattering regime," J. Acoust. Soc. Am. 90, 1742-1748.

Froment, B. (2011). "Monitoring slight mechanical changes using seismic background noise," Ph.D. thesis, University of Grenoble, France.

Grêt, A., Snieder, R., Aster, R. C., and Kyle, P. R. (2005). "Monitoring rapid temporal change in a volcano with coda wave interferometry," Geophys. Res. Lett. 32, L06304, doi:10.1029/2004GL021143.

Hansen, P. (1992). "Analysis of discrete ill-posed problems by means of the L-curve,” SIAM Rev. 34, 561-580.

Lagendijk, A., and Van Tiggelen, B. (1996). "Resonant multiple scattering of light," Phys. Rep. 270, 143-215.

Larose, E., Planes, T., Rossetto, V., and Margerin, L. (2010). "Locating a small change in a multiple scattering environment,” Appl. Phys. Lett. 96, 204101.

Meunier, J., Huguet, F., and Meynier, P. (2001). "Reservoir monitoring using permanent sources and vertical receiver antennae: The céré-la-ronde case study," The Leading Edge 20, 622-629.

Nieuwenhuizen, T., and van Rossum, M. (1993). "Role of a single scatterer in a multiple scattering medium," Phys. Lett. A 177, 102-106.

Nocedal, J., and Wright, S. (1999). Numerical Optimization (Springer, New York), pp. 1-636.

Obermann, A., Froment, B., Campillo, M., Larose, E., Planès, T., Valette, B., Chen, J., and Liu, Q. (2014). "Seismic noise correlations to image structural and mechanical changes associated with the Mw7.9 2008 Wenchuan earthquake,” J. Geophys. Res. 19, 3155-3168.

Obermann, A., Planès, T., Larose, E., and Campillo, M. (2013). "Imaging preeruptive and coeruptive structural and mechanical changes of a volcano with ambient seismic noise," J. Geophys. Res. 118, 1-10, doi:10.1002/ 2013 JB010932.

Paasschens, J. C. J. (1997). "Solution of the time-dependent Boltzmann equation,” Phys. Rev. E 56, 1135-1141.

Pacheco, C., and Snieder, R. (2005). "Time-lapse travel time change of multiply scattered acoustic waves," J. Acoust. Soc. Am. 118, 1300-1310.

Page, J., Cowan, M., and Weitz, D. (2000). "Diffusing acoustic wave spectroscopy of fluidized suspensions," Phys. B (Amsterdam, Neth.) 279, 130-133.

Pine, D., Weitz, D., Chaikin, P., and Herbolzheimer, E. (1988). "Diffusing wave spectroscopy," Phys. Rev. Lett. 60, 1134-1137.

Planès, T. (2013). "Imaging local changes in the multiple scattering regime," Ph.D. thesis, University of Grenoble, France.

Planès, T., and Larose, E. (2013). "A review of ultrasonic coda wave interferometry in concrete," Cem. Concr. Res. 53, 248-255.

Planès, T., Larose, E., Margerin, L., Rossetto, V., and Sens-Schönfelder, C. (2014). "Decorrelation and phase-shift of coda waves induced by local changes: Multiple scattering approach and numerical validation," Waves Random Complex Media 24, 99-125.

Poupinet, G., Ellsworth, W., and Frechet, J. (1984). "Monitoring velocity variations in the crust using earthquake doublets: An application to the Calaveras Fault, California," J. Geophys. Res. 89, 5719-5731, doi:10.1029/JB089iB07p05719.

Rakotonarivo, S. T., Walker, S. C., Kuperman, W. A., and Roux, P. (2011). "Localization of a small change in a multiple scattering environment without modeling of the actual medium," J. Acoust. Soc. Am. 130, 3566-3573.

Ratdomopurbo, A., and Poupinet, G. (1995). "Monitoring a temporal change of seismic velocity in a volcano: Application to the 1992 eruption of Mt. Merapi (Indonesia)," Geophys. Res. Lett. 22, 775-778, doi:10.1029/95GL00302.

Rossetto, V., Margerin, L., Planès, T., and Larose, E. (2011). "Locating a weak change using diffuse waves: Theoretical approach and inversion procedure," J. Appl. Phys. 109, 034903.

Ryzhik, L., Papanicolaou, G., and Keller, J. B. (1996). "Transport equations for elastic and other waves in random media," Wave Motion 24, 327-370.

Sato, H. (1993). "Energy transportation in one- and two-dimensional scattering media: Analytic solutions of the multiple isotropic scattering model," Geophys. J. Int. 112, 141-146.

Sens-Schönfelder, C., and Wegler, U. (2006). "Passive image interferometry and seasonal variations of seismic velocities at Merapi Volcano, Indonesia," Geophys. Res. Lett. 33, L21302, doi:10.1029/2006GL027797.

Shang, T., and Gao, L. (1988). "Transportation theory of multiple scattering and its application to seismic coda waves of impulsive source," Scientia Sinica 31, 1503-1514.

Snieder, R. (2006). "The theory of coda wave interferometry," Pure Appl. Geophys. 163, 455-473.

Taflove, A., and Hagness, S. (2005). Computational Electrodynamics: The Finite-Difference Time-Domain Method (Artech House, Norwood, MA), pp. 1-1006.

Tarantola, A. (2005). Inverse Problem Theory and Methods for Model Parameter Estimation (Society for Industrial Mathematics, Philadelphia, PA), pp. 1-342.

Tarantola, A., and Valette, B. (1982). "Generalized nonlinear inverse problems solved using the least squares criterion,” Rev. Geophys. Space Phys. 20, 219-232, doi:10.1029/RG020i002p00219.

Vanneste, C., Feng, S., and Sornette, D. (1993). "Nondestructive evaluations in multiple scattering media," J. Acoust. Soc. Am. 93, 2311-2312.

van Rossum, M., and Nieuwenhuizen, T. (1999). "Multiple scattering of classical waves: Microscopy, mesoscopy, and diffusion," Rev. Mod. Phys. 71, 313-371.

Vergely, J.-L., Valette, B., Lallement, R., and Raimond, S. (2010). "Spatial distribution of interstellar dust in the Sun's vicinity: Comparison with neutral sodium-bearing gas," Astron. Astrophys. 518, A31.

Wegler, U., and Lühr, B.-G. (2001). "Scattering behaviour at Merapi Volcano (Java) revealed from an active seismic experiment," Geophys. J. Int. 145, 579-592.

Wegler, U., and Sens-Schönfelder, C. (2007). "Fault zone monitoring with passive image interferometry,” Geophys. J. Int. 168, 1029-1033. 